\title{
Expression of interleukin-6 is associated with epithelial-mesenchymal transition and survival rates in gallbladder cancer
}

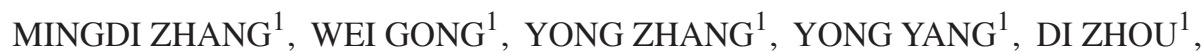 \\ MINGZHE WENG $^{1}$, YIYU QIN ${ }^{1}$, ALEX JIANG $^{2}$, FEI MA ${ }^{3}$ and ZHIWEI QUAN ${ }^{1}$
}

\author{
${ }^{1}$ Department of General Surgery, Xinhua Hospital, Shanghai Jiaotong University, School of Medicine, Shanghai 200092, \\ P.R. China; ${ }^{2}$ Department of Health Science, Schulich School of Medicine and Dentistry, Western Ontario University, London, \\ ON N6A 3K6, Canada; ${ }^{3}$ Department of Oncology, Xinhua Hospital, Shanghai Jiaotong University, \\ School of Medicine, Shanghai 200092, P.R. China
}

Received February 28, 2014; Accepted November 19, 2014

DOI: $10.3892 / \mathrm{mmr} .2014 .3143$

\begin{abstract}
The present study aimed to investigate the expression of interleukin-6 (IL-6) in gallbladder cancer (GBC) tissues and its correlation with survival rate. The association between IL-6 and epithelial-mesenchymal transition (EMT)-associated markers was also examined. Using immunohistochemistry, reverse transcription quantitative polymerase chain reaction (RT-qPCR) and western blot analysis, the protein and mRNA expression levels of IL-6, Twist, E-cadherin and Vimentin in 20 GBC tissues were analyzed. The IL-6, Twist and Vimentin proteins were overexpressed in 40, 20 and $70 \%$ of the human GBC samples, respectively. The protein expression of E-cadherin was higher in only $5 \%$ of the GBC samples. These differences were significant $(\mathrm{P}<0.05)$. Western blot analysis also revealed overexpression of IL-6, Twist and Vimentin and underexpression of E-cadherin in the GBC samples with poor differentiation, local invasion and a higher tumor-node-metastasis (TNM) stage $(\mathrm{P}<0.05)$. Higher mRNA expression levels of IL-6, Twist and Vimentin and a reduced expression level of E-cadherin were also demonstrated in the GBC tissues $(\mathrm{P}<0.05)$. The degree of differentiation, local invasion, lymph node metastasis and clinical stage were significantly associated with the mRNA expression levels of IL-6, Twist and E-cadherin. The increased
\end{abstract}

Correspondence to: Professor Zhiwei Quan, Department of General Surgery, Xinhua Hospital, Shanghai Jiaotong University, School of Medicine, 1665 Kongjiang Road, Shanghai 200092, P.R. China

E-mail: zhiwquan@163.com

Professor Fei Ma, Department of Oncology, Xinhua Hospital, Shanghai Jiaotong University, School of Medicine, 1665 Kongjiang Road, Shanghai 200092, P.R. China

E-mail: irismafei@126.com

Key words: interleukin-6, epithelial-mesenchymal transition, gallbladder cancer, metastasis, survival expression levels of IL-6 and Twist and the reduced expression of E-cadherin correlated with shorter median survival rates $(\mathrm{P}<0.05)$. Line regression results revealed correlation among the mRNA expression levels of IL-6, Twist, E-cadherin and Vimentin. To the best of our knowledge, the present study is the first to demonstrate that IL-6 is associated with EMT-associated markers, tumor differentiation, local invasion, TNM stage and survival rates in GBC.

\section{Introduction}

Gallbladder cancer (GBC) is a relatively rare type of neoplasm, but is particularly life-threatening. It is the most common biliary tract tumor and the seventh most common type of malignancy of the digestive tract worldwide (1). As the clinical symptoms are subtle, the majority of patients are diagnosed at an advanced stage. The 5 year mortality rate is up to $90 \%$ (2), while the median survival prognosis for patients is 4-6 months. Due to the advanced stage at presentation, only a third of patients are potential candidates for surgery (3). GBC is highly invasive and spreads to regional lymph nodes at an early stage. In addition, it has a high rate of recurrence (4). Treatment with adjuvant therapy has been considered, however, no previous studies have provided conclusive evidence supporting the benefit of adjuvant treatment for GBC (5). Thus, the majority of patients present with metastasis at the time of diagnosis.

GBC is suspected in patients with a long history of chronic cholecystitis secondary to cholelithiasis who demonstrate a change in symptoms. Interleukin-6 (IL-6) is a pleiotropic cytokine involved in acute inflammation, hematopoiesis $(6,7)$ and the proliferation of cancer cells (8). Increased expression of IL-6 has been detected and associated with an unfavorable prognosis and metastasis in patients with cancer (9). Therefore, targeting IL-6-mediated pathways can offer an effective treatment modality (10). Several studies have suggested the role of IL-6 in modulating the tumor microenvironment, which is triggered by inducing epithelial-to mesenchymal transition (EMT) followed by downregulation the expression of E-cadherin and upregulation the expression levels of Vimentin, $\mathrm{N}$-cadherin, 
Snail and Twist $(11,12)$. EMT is an important mechanism in tumor invasion and metastasis (13) and Twist is important in promoting EMT (14). However, the exact effect of the expression of IL-6 remains to be elucidated.

The present study examined the association between IL-6, Twist, EMT and GBC. The progression, invasion and metastasis of GBC was analyzed by investigating the expression of the epithelial marker E-cadherin and interstitial marker Vimentin. The results may improve understanding of GBC prognosis and targeted therapy.

\section{Materials and methods}

Clinical specimens. Human GBC tissues were obtained with informed consent from the Eastern Hepatic Biliary Hospital affiliated with the Second Military Medicine University (Shanghai, China) and the procedures used in the present study were approved by the Protection of Human Subjects Committee of the Eastern Hepatic Biliary Hospital affiliated with the Second Military Medicine University. A total of $20 \mathrm{GBC}$ specimens and their surrounding tissues were obtained from patients who underwent cholecystectomy. Immediately following surgical removal, half of the tissues were snap-frozen in liquid nitrogen for storage and the remaining half were fixed in $4 \%$ paraformaldehyde (DingGuo Biotech Co., Ltd, Shanghai, China) and embedded in paraffin (DingGuo Biotech Co., Ltd).

Immunohistochemical staining. The samples, prepared from the paraffin-embedded block, were rehydrated and then incubated in $3 \%$ hydrogen peroxide for $15 \mathrm{~min}$ to block endogenous peroxidase. For antigen retrieval, the samples were boiled in a pressure cooker for $10 \mathrm{~min}$. Nonspecific binding was inhibited with $10 \%$ normal goat serum (Boshide Biological Engineering, Co., Ltd, Wuhan, China) for $20 \mathrm{~min}$ at $37^{\circ} \mathrm{C}$. The samples were then incubated at $4^{\circ} \mathrm{C}$ overnight with the following primary antibodies: Rabbit anti-mouse polyclonal IL-6 (1:50; cat. no. ab6672; Abcam, Cambridge, MA, USA), mouse anti-goat polyclonal Twist (1:50; cat. no. ab50887; Abcam), rabbit anti-mouse polyclonal E-cadherin (1:100; sc-7870; Santa Cruz Biotechnology, Inc., Santa Cruz, CA, USA) and rabbit anti-mouse polyclonal Vimentin (1:100; cat. no. sc-5565; Santa Cruz Biotechnology, Inc.). The sections were treated with goat anti-rabbit/anti-mouse polyclonal secondary antibodies conjugated to horseradish peroxidase (Abcam, Cambridge, MA, USA) for $30 \mathrm{~min}$ at room temperature and stained with diaminobenzidine (Beyotime Institute of Biotechnology, Shanghai, China) until brown granules appeared. The sections were then counterstained with hematoxylin (DingGuo Biotech Co., Ltd) for $2 \mathrm{~min}$ at room temperature.

Evaluation of immunohistochemical staining. The sections were evaluated by two pathologists in a blinded-manner using a light microscope (DMI3000B; Leica Microsystems AG, Solms, Germany). A semi-quantitative scoring criterion for immunohistochemistry was used, in which expression was determined based on the percentage of positive cells and staining intensity. The scores were interpreted as shown in Table I.

The final score was determined by the expression rate and intensity of proteins, graded as '-' for 0 point, '+' for 1-2 points, '++' for 3-4 points and ' +++ ' for 5-6 points. Immunoreactivity
' \pm ' was used to denote overexpression and '++/+++' was used to denote underexpression for statistical analysis.

Reverse transcription quantitative polymerase chain reaction (RT-qPCR). Total RNA was extracted from $100 \mathrm{mg}$ GBC tissues and the surrounding tissues. The tissues were added to $1 \mathrm{ml}$ TRIzol reagent (Takara Bio, Inc., Tokyo, Japan) and homogenized according to the manufacturer's instructions. The first strand of cDNA was synthesized from $500 \mathrm{ng}$ total RNA using PrimeScript ${ }^{\circledR}$ Reverse Transcriptase (Takara Bio, Inc.). The qPCR was performed in a reaction volume of $20 \mu \mathrm{l}$, including $2 \mu \mathrm{l}$ cDNA. The primer sequences used are shown in Table II.

The PCR conditions were as follows: $95^{\circ} \mathrm{C}$ for $30 \mathrm{sec}$, followed by 40 cycles of $95^{\circ} \mathrm{C}$ for $5 \mathrm{sec}$ and $60^{\circ} \mathrm{C}$ for $34 \mathrm{sec}$. The relative quantification of genes was analyzed using the comparative threshold cycle $\left(\mathrm{C}_{t}\right)$ method. To ensure that only a specific band was produced, melting curve analysis was performed at the end of each PCR experiment. The term $-\Delta C_{t}$ was used to describe the expression level of mRNA. The expression was subsequently divided into lower expression and higher expression groups, based on whether the mRNA levels were above or below the mean value.

Western blot analysis. Western blot analysis was performed, as previously described (15). For the total protein extraction, $100 \mathrm{mg}$ of frozen tissue samples, previously stored in liquid nitrogen, were ground and homogenized using radioimmunoprecipitation assay lysis buffer (Beyotime Institute of Technology). The total protein concentrations of the cell extracts were determined using a bicinchoninic acid assay system (Beyotime Institute of Biotechnology) with bovine serum albumin as the standard. For electrophoresis, $80 \mu \mathrm{g}$ of the total protein was added to each lane on SDS-PAGE gels (8, 10 and 15\%; Beyotime Institute of Biotechnology) and the protein was then blotted onto a polyvinylidene difluoride membrane (DingGuo Biotech Co., Ltd) by wet transfer (200 mA, 1-2 h). The membranes were inhibited with $5 \%$ skimmed milk and incubated with anti-IL-6 (cat. no. ab6672; Abcam, US), anti-Twist (cat. no. ab50887; Abcam), anti-E-cadherin (cat. no. sc-7870; Santa Cruz Biotechnology, Inc.), anti-Vimentin (cat. no. sc-5565; Santa Cruz Biotechnology, Inc.) and anti- $\beta$-actin antibodies (cat. no. \#4970; Cell Signaling Technology, Inc.), respectively, at $4^{\circ} \mathrm{C}$ overnight. This was followed by incubation with goat anti-rabbit/anti-mouse secondary antibody conjugated to horseradish peroxidase $(1: 1,000)$. The stain was visualized using an enhanced chemiluminescent (ECL) detection reagent from Millpore (Rockford, IL, USA). Images were captured and the optical densities of the bands were quantified using a Gel Doc 2000 system (BioRad, Hercules, CA, USA).

Statistical analysis. The results of all the assays are expressed as the mean \pm standard deviation. All the assays were performed independently in triplicate. The data were analyzed using Prism 5.0 software (GraphPad Software, Inc., La Jolla, CA, USA). The survival curves were generated using the Kaplan-Meier method. The significance of the observed differences were determined using Student's t-test 
Table I. Immunohistochemical staining score system.

\begin{tabular}{ll}
\hline Score & \multicolumn{1}{c}{ Description } \\
\hline 0 & $\leq 5 \%$ of positive cells or negative staining \\
1 & $5-25 \%$ of positive cells or weak staining \\
2 & $25-50 \%$ of positive cells or moderate staining \\
3 & $50 \%$ of positive cells or strong staining
\end{tabular}

Table II. Primer sequences.

\begin{tabular}{lll}
\hline Gene & Forward/Reverse & Sequence $\left(5^{\prime}-3^{\prime}\right)$ \\
\hline$\beta$-actin & Forward & CTGGGACGACATGGAGAAAA \\
Interleukin-6 & Reverse & AAGGAAGGCTGGAAGAGTGC \\
& Forward & CCACACAGACAGCCACTCAC \\
Twist & Reverse & GATGATTTCACCAGGCAAGTC \\
& Forward & AGTCCGCAGTCTTACGAGGAG \\
E-cadherin & Reverse & GACCTGGTAGAGGAAGTCGATG \\
& Forward & GTCTCTCTCACCACCTCCACAG \\
Vimentin & Reverse & CTCGGACACTTCCACTCTCTTT \\
& Forward & GAAGAGAACTTTGCCGTTGAAG \\
& Reverse & GAAGGTGACGAGCCATTTC \\
\hline
\end{tabular}

A

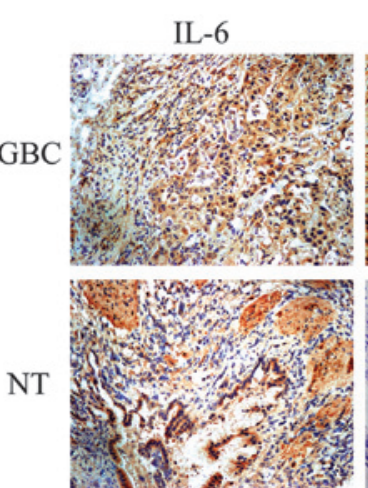

Twist
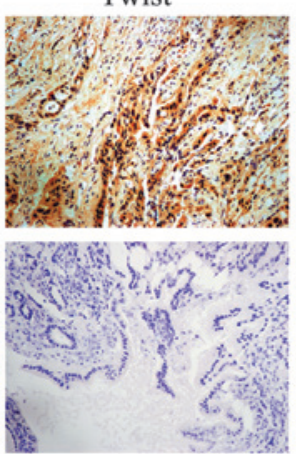

E-cadherin

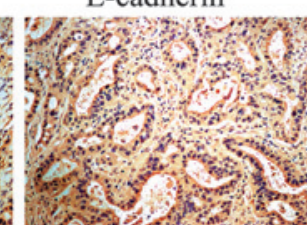

Vimentin
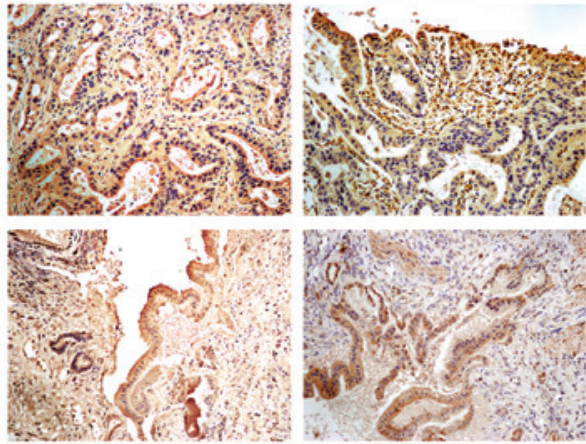

B

\begin{tabular}{ccccccccc}
\hline \multirow{2}{*}{ Marker } & \multirow{2}{*}{ Group } & Cases & \multicolumn{7}{c}{ Expression of protein } & & \multirow{2}{*}{$\boldsymbol{\chi 2}$} & \multirow{2}{*}{$\boldsymbol{P}$} \\
& & & - & + & ++ & +++ & & \\
\hline \multirow{2}{*}{ IL-6 } & Adjacent tissue & 20 & 11 & 7 & 2 & 0 & \multirow{2}{*}{8.329} & 0.040 \\
& Cancer tissue & 20 & 4 & 8 & 6 & 2 & & \\
\multirow{2}{*}{ Twist } & Adjacent tissue & 20 & 14 & 5 & 1 & 0 & \multirow{2}{*}{6.918} & 0.031 \\
& Cancer tissue & 20 & 6 & 10 & 4 & 0 & & \\
\multirow{5}{*}{ E-cadherin } & Adjacent tissue & 20 & 5 & 10 & 3 & 2 & \multirow{2}{*}{9.957} & 0.019 \\
& Cancer tissue & 20 & 14 & 5 & 1 & 0 & & \\
& Adjacent tissue & 20 & 8 & 7 & 3 & 2 & \multirow{2}{*}{8.455} & 0.037 \\
\hline
\end{tabular}

Figure 1. Expression levels of IL-6 protein and epithelial-mesenchymal transition-associated proteins in GBC tissues and in paired adjacent tissues. (A) Representative immunostaining of IL-6, Twist, E-cadherin and Vimentin in the GBC and adjacent tissues (magnification, x200). (B) Different protein expression levels of IL-6, Twist, E-cadherin and Vimentin in 20 paired samples graded based on protein expression intensity as: - (0 point); + (1-2 points); ++ (3-4 points) and +++ (5-6 points). GBC gallbladder cancer; NT, normal tissues; IL-6, interleukin-6.

or $\chi^{2}$ test. Associations among the IL-6, Twist, E-cadherin and Vimentin mRNA were analyzed by correlation coefficients and linear regression analysis. $\mathrm{P}<0.05$ was considered to indicate a statistically significant difference.

\section{Results}

Expression of IL-6 and EMT-associated protein in the GBC tissues. The expression levels of IL- 6 and the Twist, E-cadherin 


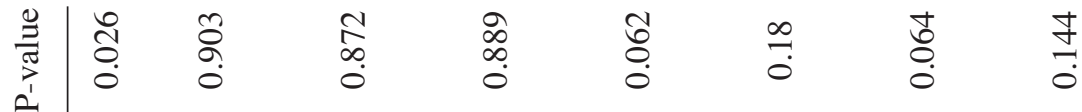

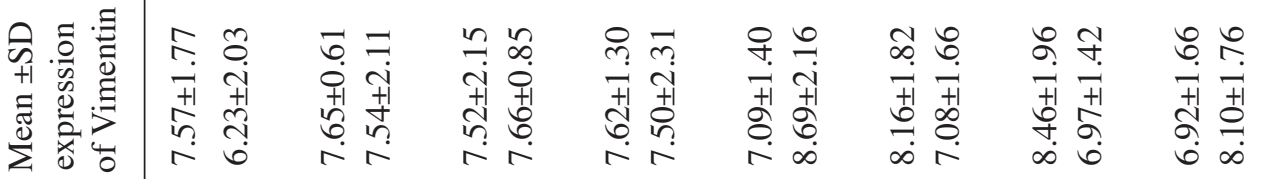

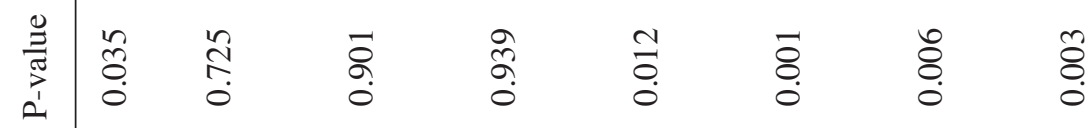

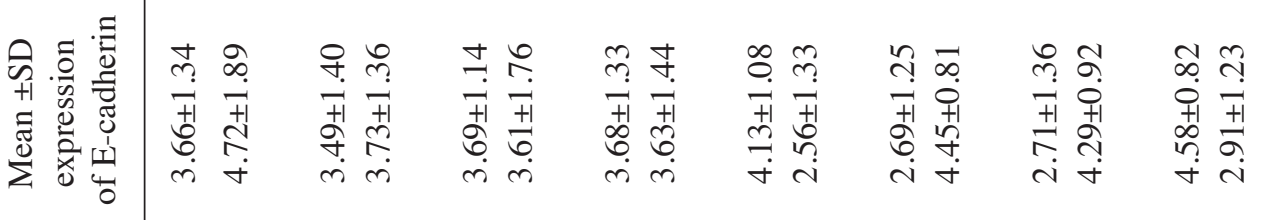

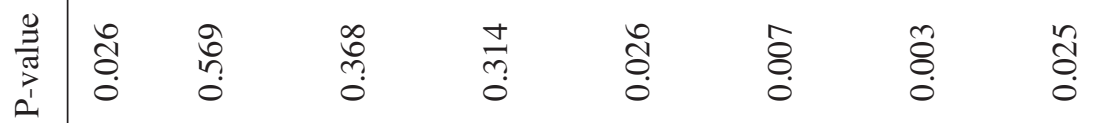

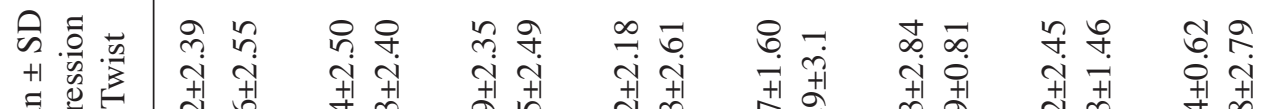

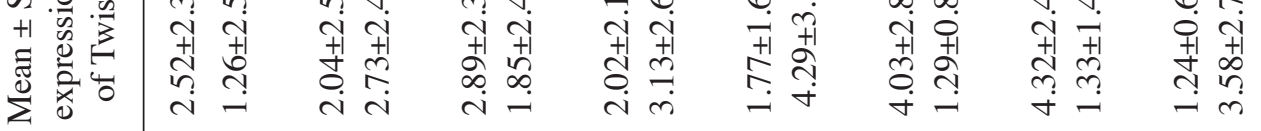

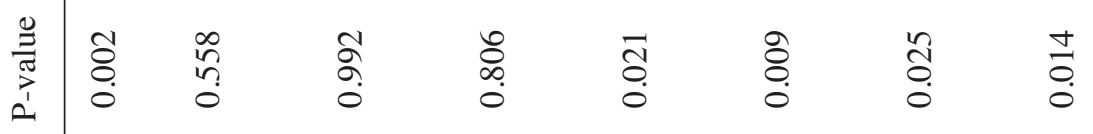

命泀

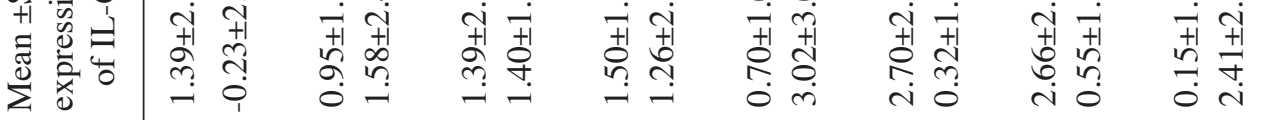

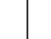

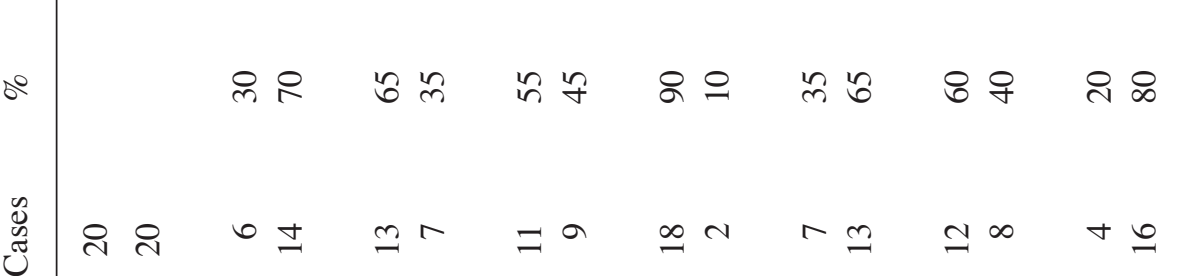


and Vimentin EMT-associated proteins were determined in 20 human GBC tissues and 20 surrounding tissues using immunohistochemistry (Fig. 1A). The results revealed overexpression of IL-6, Twist and Vimentin and underexpression of E-cadherin in the GBC tissues compared with the adjacent tissues (Fig. 1A). The results also revealed that the IL-6, Twist and Vimentin proteins were overexpressed in 8, 4 and 14 (40, 20 and $70 \%$ ) of the GBC samples, respectively. The underexpression of E-cadherin was observed in a single GBC sample $(5 \%)$. By contrast, the expression levels of IL-6, Twist, Vimentin in the GBC tissues were significantly higher compared with those in the surrounding tissues $\left(\chi^{2}=8.329, \mathrm{P}=0.040 ; \chi^{2}=6.918\right.$, $\mathrm{P}=0.031$ and $\chi^{2}=8.455, \mathrm{P}=0.037$, respectively). However, the expression of E-cadherin was significantly lower in the GBC tissues $\left(\chi^{2}=9.957, \mathrm{P}=0.019\right)$.

Western blot analysis also revealed the overexpression of IL-6, Twist and Vimentin and underexpression of E-cadherin in the GBC tissues compared with the normal adjacent tissues ( $\mathrm{P}<0.05$; Fig. 2Aa and $\mathrm{b})$. The decrease in the differentiation level of the GBC tissue (Fig. 2Ac and d), increase in tumor-node-metastasis (TNM) stage (Fig. 2Ae and f) and positive local invasion (Fig. 2A, g and h) were correlated with increased expression levels of IL-6, Twist and Vimentin $(\mathrm{P}<0.05)$. The expression of E-cadherin in the GBC tissues was significantly lower compared with that in the surrounding tissues $(\mathrm{P}<0.05)$ and was lower in patients with GBC exhibiting high grade differentiation, local invasion and a high TNM stage ( $\mathrm{P}<0.05$; Fig. $2 \mathrm{~A})$.

IL-6 and EMT-associated mRNA expression in gastric cancer tissues is associated with advanced clinical stage, lymph node metastasis and poor patient prognosis. Consistent with the above data, the results confirmed that the expression levels of IL-6, Twist and Vimentin in the GBC tissues were significantly higher ( $\mathrm{P}<0.05$; Fig. 3A, B and D). However, the mRNA expression of E-cadherin was significantly lower in the GBC tissues $(\mathrm{P}=0.0265$; Fig. 3C). The correlations between the IL-6 and EMT-associated mRNA expression and the clinicopathologic characteristics of GBC are summarized in Table III. The differentiation, local invasion, lymph node status and clinical stages were correlated with the expression of IL-6. The median expression level of IL- 6 was $2.41 \pm 2.21$ in the 20 cases with advanced stage (stage III and IV) and 0.15 $\pm 1.20(\mathrm{P}=0.014)$ in cases with early-stage (stage I and II) disease. In the 20 cases of GBC with either local invasion or lymph node metastasis, the median expression levels of IL-6 were $2.70 \pm 2.36$ and $2.66 \pm 2.58$, respectively. This was significantly higher compared with the expression levels in the 20 adjacent tissues $(0.32 \pm 1.14$; $\mathrm{P}=0.009$ and $0.55 \pm 1.27 ; \mathrm{P}=0.025$, respectively). The expression of IL- 6 in the GBC patients did not correlate with gender, age or tumor size. A statistically significant correlation was observed between the degree of differentiation, local invasion, lymph node metastasis, clinical stage and Twist/E-cadherin expression. However, no statistically significant correlation was observed between the expression of Vimentin and the clinicopathologic characteristics. In addition, the present study examined whether the mRNA expression levels of Il-6, Twist, E-cadherin and Vimentin were associated with survival rate in patients with GBC. Based on the mean expression level of IL-6 (1.394), Twist (2.524), E-cadherin (3.660) and Vimentin (7.568), as shown in Fig. 3A-D, the GBC specimens were divided into a higher and a
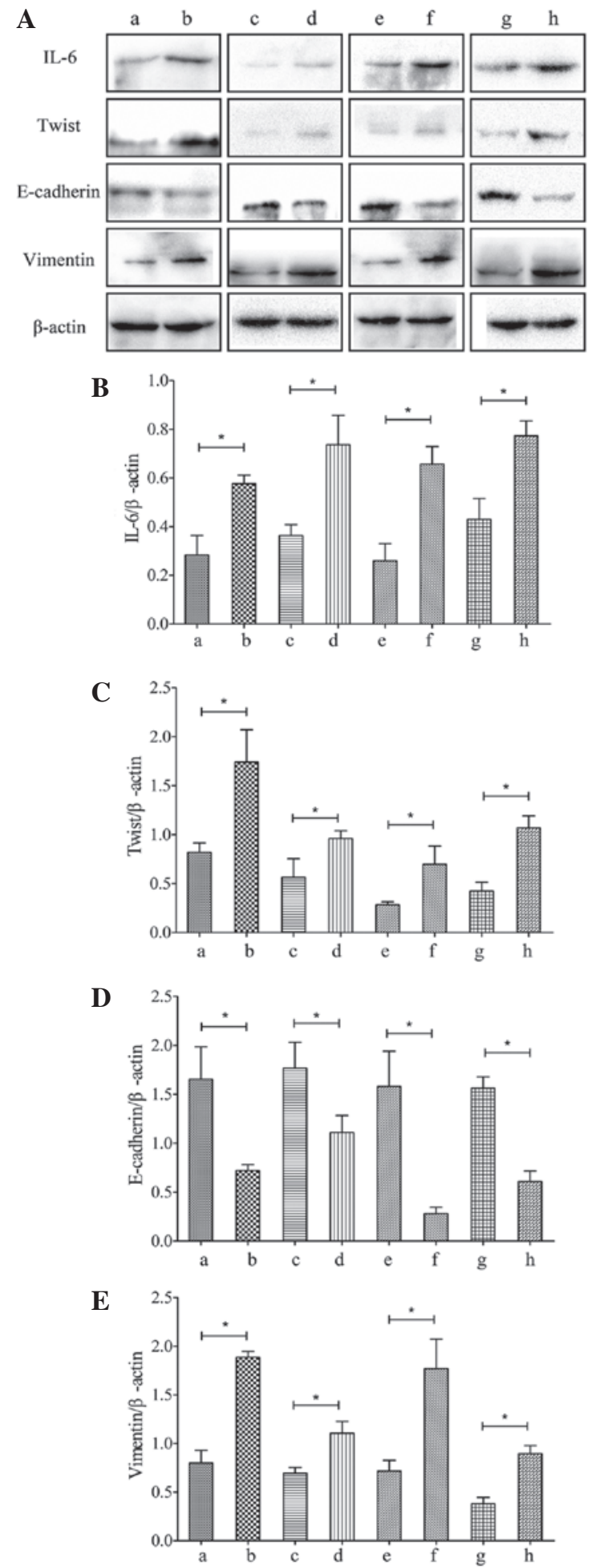

Figure 2. (A) Protein expression levels of IL-6, Twist, E-cadherin and Vimentin in gallbladder cancer tissues. (B-E) Relative protein expression levels of IL-6, Twist, E-cadherin and Vimentin in gallbladder cancer tissues. a, adjacent tissue; b, gallbladder cancer tissue; c, well and moderately differentiated; d, poorly differentiated; e, TNM stage I-II; f, TNM stage III-IV; g, without local invasion; h, local invasion. Values are presented as the mean \pm standard deviation. ${ }^{*} \mathrm{P}<0.05$. IL-6, interleukin-6; TNM, tumor-node-metastasis.

lower expression group. Kaplan-Meier survival analyis revealed that patients whose tumors exhibited increased expression of IL-6 or Twist compared with that of the lower expression group or reduced expression of E-cadherin compared with that of the 
A

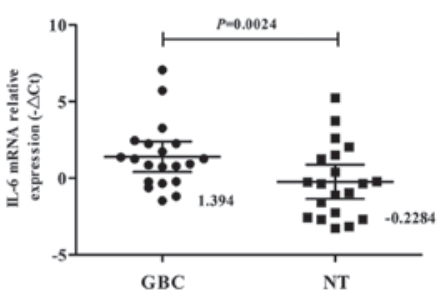

C

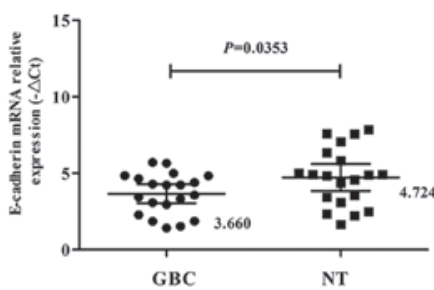

B

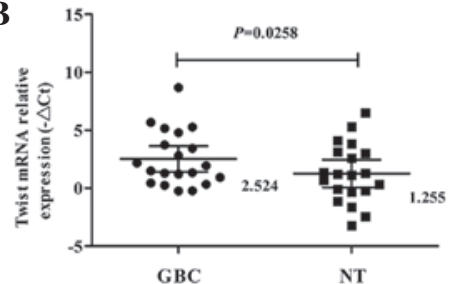

D

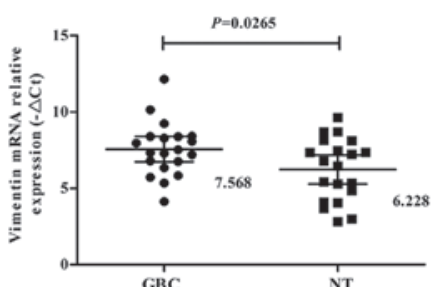

$\mathbf{E}$

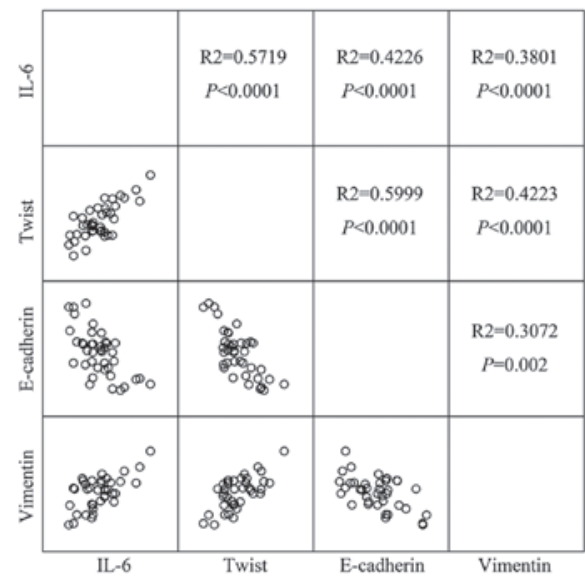

Figure 3. Results of the expression analysis of IL-6, Twist, E-cadherin and Vimentin in 20 matched GBC tissues and adjacent tissues. (A-D) mRNA expression levels of IL-6, Twist, E-cadherin and Vimentin in GBC tissues and in adjacent normal tissues. (E) Scatter plot analysis of all the GBC and NT samples of IL-6, Twist, E-cadherin and Vimentin with $\mathrm{R}^{2}$ and P-values. IL-6, interleukin-6; GBC, gall bladder cancer; NT, normal tissue.
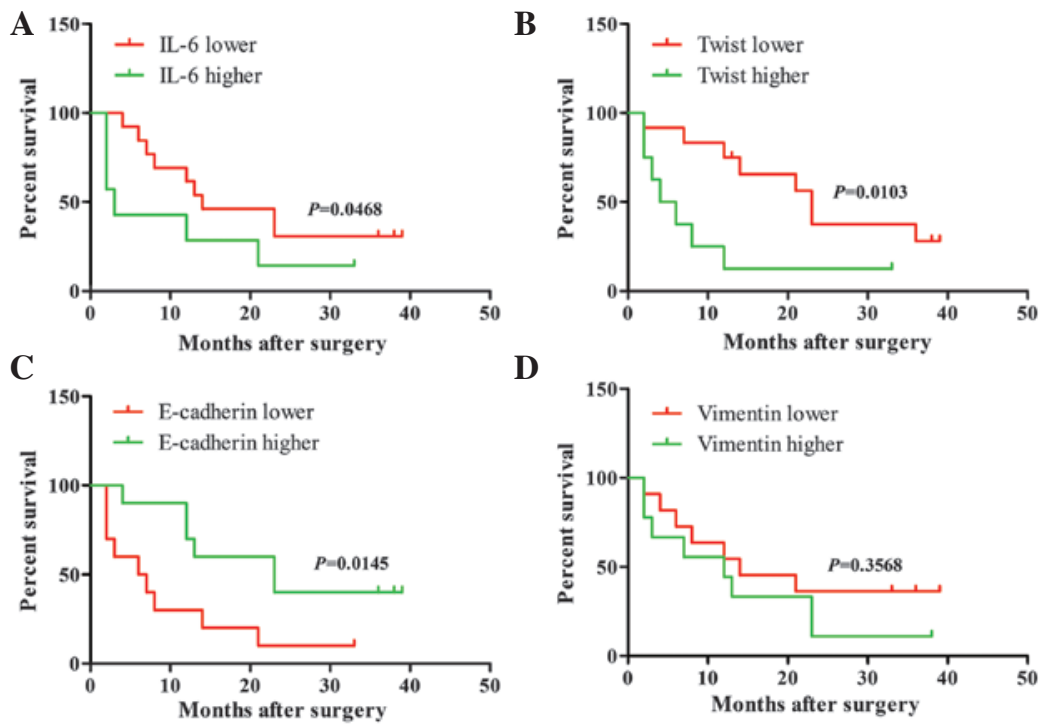

Figure 4. Comparison of Kaplan-Meier survival curves for gallbladder cancer patients between higher and lower expression levels of (A) IL-6, (B) Twist, (C) E-cadherin and (D) Vimentin. IL-6, interleukin-6.

higher expression group had a shorter median survival rates at $10.71 \pm 12.19,8.75 \pm 10.38$ and $9.8 \pm 10.20$ months, respectively ( $\mathrm{P}=0.0486, \mathrm{P}=0.0103$ and $\mathrm{P}=0.0145$; Fig. 4A-D).
IL-6 is associated with the expression of twist and can accurately discriminate between $G B C$ and adjacent tissues. Line regression results produced $\mathrm{R}^{2}$ values to compare the mRNA 
expression levels of IL-6, Twist, E-cadherin and Vimentin, respectively, in the GBC and adjacent tissues. Significant correlations were observed among these four mRNAs $(\mathrm{P}<0.05 ;$ Fig. $3 \mathrm{E})$.

\section{Discussion}

The present study demonstrated theat IL- 6 protein and mRNA were overexpressed in GBC tissues (Figs. 1-3) and IL-6, Twist and E-cadherin were associated with local invasion, lymph node metastasis, poor differentiation and poor clinical prognosis in GBC. This is the first study, to the best of our knowledge, to examine the correlation between IL-6 and EMT and prognosis.

Although the mRNA expression of IL- 6 was increased in the $20 \mathrm{GBC}$ tissues, its source remains to be elucidated. Several cancer patients exhibit increased serum levels of IL-6, which can originate from a number of sources, including tumor cells and macrophages (16). If cancer cells increasingly secrete IL-6, it may act in an autocrine manner to enhance the metastatic ability (17) and resistance of the tumor to treatment (18). The increased levels of IL-6 have been correlated with poor prognosis and survival rate in a variety of types of cancer (19-22). Previous studies on solid tumors, including gastric, renal cell, colorectal, prostate, non-small cell lung, melanoma and head and neck cancer and hematologic malignancies, including myeloma and non-Hodgkin's lymphoma, have indicated the potential prognostic significance of IL-6 levels (19-22).

IL-6 signaling activates STAT3 (23), which is required for malignant transformation. It also has multiple protumorigenic functions, including the promotion of tumor cell proliferation, survival, invasion, metastasis and angiogenesis $(24,25)$. IL-6 induces EMT changes in tumor cells via activation of the STAT3 signaling pathway and STAT3-knockdown reverses these changes (26). The key activity of EMT (27) is hypothesized to be the reduction of cell-to-cell adhesion and induction of cell motility through downregulation of E-cadherin and may be associated with the expression of Twist and Vimentin (14).

Consistent with the data of the present study, certain studies have identified that IL-6 induces an EMT phenotype $(11,12)$. Additionally, increased expression of Twist and reduced expression of E-cadherin are correlated with poor differentiation and local invasion. Twist and E-cadherin are statistically significant prognostic factors in several types of cancer (28-32).

In the present study, linear correlation analysis revealed that the mRNA expression of IL-6 was positively correlated with the EMT-associated markers (Fig. 3E). These results suggested that the four genes have synergistic effects in tumorgenesis and metastasis in GBC. It also indicated that the malignant transformation process of GBC is accompanied by EMT.

In conclusion, the expression of IL-6 correlated with EMT-associated mRNA and protein expression, local invasion, lymph node metastasis, shorter survival time, poor clinical stage and differentiation. GBC is often diagnosed at an advanced stage and is associated with poor prognosis. It is possible to downregulate the expression of IL-6 through adjuvant therapy and several clinical studies have supported the use of IL-6 as a therapeutic target $(33,34)$. However, further studies are required to fully define the association between IL-6 and EMT in GBC.

\section{Acknowledgements}

This study was supported by the Foundation of Shanghai Jiaotong University School of Medicine (no. 12XJ22004) the Shanghai Science and Technology Bureau Introductory Project (no. 124119a0600) and the National Natural Science Foundation of China (no. 81272747).

\section{References}

1. Ferlay J, Shin HR, Bray F, Forman D, Mathers C and Parkin DM: GLOBOCAN 2008v2.0: Cancer incidence and mortality worldwide. IARC CancerBase No. 10. Lyon, France: International Agency for Research on Cancer, 2010. http://globocan.iorc.fr. (Accessed August 2012).

2. Alvi AR, Siddiqui NA and Zafar H: Risk factors of gallbladder cancer in Karachi-a case-control study. World J Surg Oncol 9: 164,2011

3. Reid KM, Ramos-De la Medina A and Donohue JH: Diagnosis and surgical management of gallbladder cancer: a review. J Gastrointest Surg 11: 671-681, 2007.

4. Hundal R and Shaffer EA: Gallbladder cancer: epidemiology and outcome. Clin Epidemiol 6: 99-109, 2014.

5. Jayaraman S and Jarnagin WR: Management of gallbladder cancer. Gastroenterol Clin North Am 39: 331-342, 2010.

6. Kishimoto T: Interleukin-6: discovery of a pleiotropic cytokine. Arthritis Res Ther 8 Suppl 2: 2, 2006.

7. Qi J, Chen N, Wang J and Siu CH: Transendothelial migration of melanoma cells involves $\mathrm{N}$-cadherin-mediated adhesion and activation of the beta-catenin signaling pathway. Mol Biol Cell 16: 4386-4397, 2005

8. Di GH, Liu Y, Lu Y, Liu J, Wu C and Duan HF: IL-6 secreted from senescent mesenchymal stem cells promotes proliferation and migration of breast cancer cells. PLoS One 9: e113572, 2014.

9. Soubrane C, Rixe O, Meric JB, Khayat D and Mouawad R: Pretreatment serum interleukin-6 concentration as a prognostic factor of overall survival in metastatic malignant melanoma patients treated with biochemotherapy: a retrospective study. Melanoma Res 15: 199-204, 2005.

10. Ataie-Kachoie P, Pourgholami MH and Morris DL: Inhibition of the IL-6 signaling pathway: a strategy to combat chronic inflammatory diseases and cancer. Cytokine Growth Factor Rev 24: 163-173, 2013.

11. Sullivan NJ, Sasser AK, Axel AE, et al: Interleukin-6 induces an epithelial-mesenchymal transition phenotype in human breast cancer cells. Oncogene 28: 2940-2947, 2009.

12. Na YR,Lee JS, Lee SJ and Seok SH: Interleukin-6-induced Twist and $\mathrm{N}$-cadherin enhance melanoma cell metastasis. Melanoma Res 23: 434-443, 2013.

13. Thiery JP: Epithelial-mesencymal transitions in tumour progression. Nat Rev Cancer 2: 442-454, 2002.

14. Liu AN, Zhu ZH, Chang SJ and Hang XS: Twist expression associated with the epithelial-mesenchymal transition in gastric cancer. Mol Cell Biochem 367: 195-203, 2012.

15. Qin Y, Zhang S, Gong W, Li J, Jia J and Quan Z: Adenovirus-mediated gene transfer of tissue factor pathway inhibitor-2 inhibits gallbladder carcinoma growth in vitro and in vivo. Cancer Sci 103: 723-730, 2012.

16. Diehl S, Anguita J, Hoffmeyer A, et al: Inhibition of Th1 differentiation by IL-6 is mediated by SOCS1. Immunity 13: $805-815,2000$

17. Luo F, Xu Y, Ling M, et al: Arsenite evokes IL-6 secretion, autocrine regulation of STAT3 signaling and miR-21 expression, processes involved in the EMT and malignant transformation of human bronchial epithelial cells. Toxicol Appl Pharmacol 273: 27-34, 2013.

18. Wang Y, Qu Y, Zhang XL, et al: Autocrine production of interleukin-6 confers ovarian cancer cells resistance to tamoxifen via ER isoforms and SRC-1. Mol Cell Endocrinol 2013. 
19. Yeh KY, Li YY, Hsieh LL, et al: Analysis of the effect of serum interleukin-6 (IL-6) and soluble IL-6 receptor levels on survival of patients with colorectal cancer. Jpn J Clin Oncol 40: 580-587, 2010.

20. Duffy SA, Taylor JM, Terrell JE, et al: Interleukin-6 predicts recurrence and survival among head and neck cancer patients. Cancer 113: 750-757, 2008.

21. Suh SY, Choi YS, Yeom ${ }^{\circ} \mathrm{CH}$, et al: Interleukin-6 but not tumour necrosis factor-alpha predicts survival in patients with advanced cancer. Support Care Cancer 21: 3071-3077, 2013.

22. Chang $\mathrm{CH}$, Hsiao $\mathrm{CF}$, Yeh YM, et al: Circulating interleukin-6 level is a prognostic marker for survival in advanced nonsmall cell lung cancer patients treated with chemotherapy. Int J Cancer 132: 1977-1985, 2013.

23. Yu H, Kortylewski M and Pardoll D: Crosstalk between cancer and immune cells: role of STAT3 in the tumour microenvironment. Nat Rev Immunol 7: 41-51, 2007.

24. Ho PL, Lay EJ, Jian W, Parra D and Chan KS: Stat3 activation in urothelial stem cells leads to direct progression to invasive bladder cancer. Cancer Res 72: 3135-3142, 2012.

25. Rathinavelu A, Narasimhan $M$ and Muthumani P: A novel regulation of VEGF expression by HIF-1 $\alpha$ and STAT3 in HDM2 transfected prostate cancer cells. J Cell Mol Med 16: 1750-1757, 2012.

26. Yadav A, Kumar B, Datta J, Teknos TN and Kumar P: IL-6 promotes head and neck tumor metastasis by inducing epithelial-mesenchymal transition via the JAK-STAT3-SNAIL signaling pathway. Mol Cancer Res 9: 1658-1667, 2011.
27. de Freitas Silva BS, Yamamoto-Silva FP, Pontes HA and Pinto Júnior DS: E-cadherin downregulation and Twist overexpression since early stages of oral carcinogenesis. J Oral Pathol Med 43: 125-31, 2013

28. Yuen HF, Chua CW, Chan YP, Wong YC, Wang X and Chan KW: Significance of TWIST and E-cadherin expression in the metastatic progression of prostatic cancer. Histopathology 50: 648-658, 2007.

29. Shibata K, Kajiyama H, Ino K, et al: Twist expression in patients with cervical cancer is associated with poor disease outcome. Ann Oncol 19: 81-85, 2008.

30. Zhao Z, Ge J, Sun Y, et al: Is E-cadherin immunoexpression a prognostic factor for head and neck squamous cell carcinoma (HNSCC)? A systematic review and meta-analysis. Oral Oncol 48: 761-767, 2012

31. Bačić B, Haller H, Mrklić I, Košta V, Carić A and Tomić S: Prognostic role of E-cadherin in patients with advanced serous ovarian cancer. Arch Gynecol Obstet 287: 1219-1224, 2013.

32. Aamodt R, Bondi J, Andersen SN, Bakka A, Bukholm G and Bukholm IR: The prognostic impact of protein expression of E-cadherin-catenin complexes differs between rectal and colon carcinoma. Gastroenterol Res Pract 2010.

33. Shinriki S, Jono H, Ota K, et al: Humanized anti-interleukin-6 receptor antibody suppresses tumor angiogenesis and in vivo growth of human oral squamous cell carcinoma. Clin Cancer Res 15: 5426-5434, 2009.

34. Guo Y, Xu F, Lu T, Duan Z and Zhang Z: Interleukin-6 signaling pathway in targeted therapy for cancer. Cancer Treat Rev 38: 904-10, 2012. 